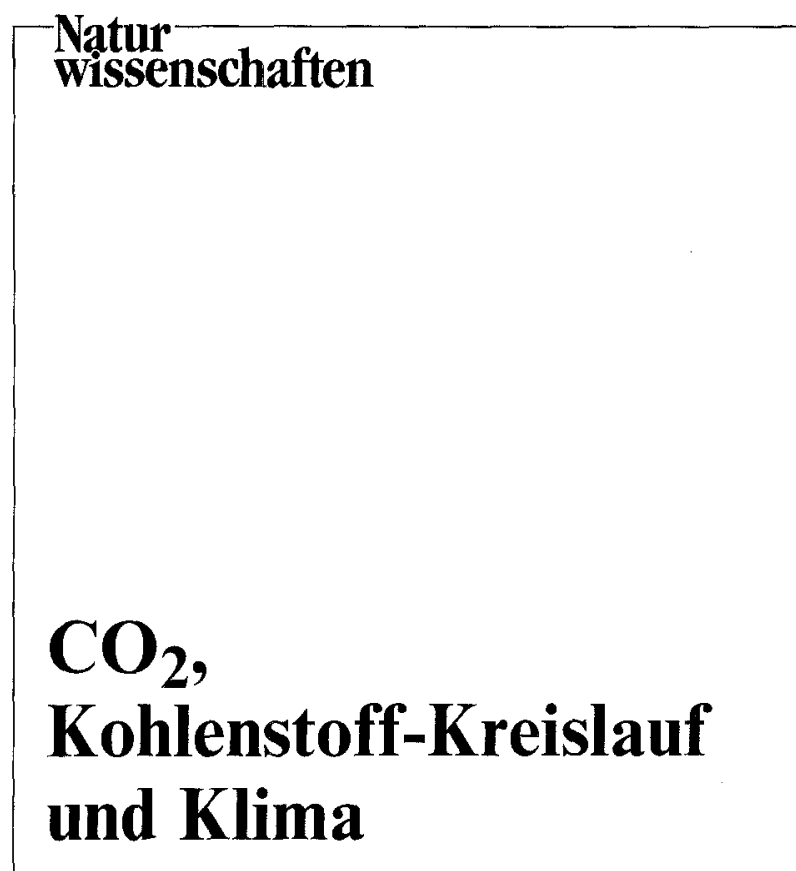

\section{Globale Kohlenstoffbilanz}

\author{
Hartmut Grass1
}

Institut für Meereskunde, D-2300 Kiel

\section{Ernst Maier-Reimer}

Max-Planck-Institut für Meteorologie, D-2000 Hamburg

\section{Egon T. Degens, Stephan Kempe} und Alejandro Spitzy

SCOPE/UNEP International Carbon Center, Geologisch-Paläontologisches Institut der Universität, D-2000 Hamburg

Past and expected emissions of anthropogenic $\mathrm{CO}_{2}$ stimulate carbon cycle and climate research. Prognoses of future $\mathrm{CO}_{2}$ levels depend on energy scenarios and on the reaction of the biosphere and hydrosphere to elevated atmospheric $\mathrm{CO}_{2}$ concentrations. The reaction of the reservoirs vegetation, freshwater and oceans to disturbances of the carbon cycle is reviewed. For the oceans first results of a simple carbon cycle model implanted in a three-dimensional general circulation model are presented. This model allows experiments not possible with previous box models.
Das $\mathrm{CO}_{2}$-Problem ist in Wissenschaft und Öffentlichkeit in den letzten zehn Jahren intensiv diskutiert worden. Es geht dabei um die Frage, ob die zunehmende Emission von anthropogenem $\mathrm{CO}_{2}$ langfristig $\mathrm{zu}$ drastischen Veränderungen des globalen Klimas führt. Seit 1958 wird unter anderen auf der Mauna-Loa-Station/Hawaii [1] der Anstieg des $\mathrm{CO}_{2}$-Partialdrucks der Luft gemessen $\left(P_{\mathrm{CO}_{2}}\right.$, Fig. 1 a). Der $P_{\mathrm{CO}_{2}}$ stieg in diesem Zeitraum exponentiell von 315 auf 340 ppmv, also um etwa $8 \% \quad(\mathrm{ppmv}=$ Volumensmischungsverhältnis in Vielfachen von $10^{-6}$ ). Eine Frage ist, warum der $\mathrm{CO}_{2}$-Gehalt nicht noch höher stieg, denn alleine der Ausstoß von $\mathrm{CO}_{2}$ bei der Energieerzeugung ist doppelt so hoch wie der beobachtete Anstieg, ganz abgesehen von möglichem zusätzlichem $\mathrm{CO}_{2}$ aus der Zerstörung der Biosphäre.

Pro Jahr werden bei der Verbrennung fossiler Kohlenstoffe 5,3 Milliarden Tonnen Kohlenstoff (Gigatonnen $=\mathrm{Gt}$ ) als $\mathrm{CO}_{2}$ in die Atmosphäre entlassen. Bisher sind schon $180 \mathrm{Gt} C$ verbraucht.

Auch aus der Biosphäre setzt der Mensch große Mengen Kohlenstoff frei. Die Rodung erst der gemäßigten (Pioniereffekt) und jetzt der tropischen Wälder, die Zerstörung der Savannen und die Abschwemmung und Oxidation der Ackerböden hat möglicherweise Kohlenstoff in gleicher Größenordnung wie die Verbrennung von Kohle, Öl und Gas mobilisiert. Moore et al. [2] und Houghton et al. [3] errechnen aus Biomasse-Modellen, daß heute zwischen 1,8 und 4,7 Gt Kohlenstoff netto pro Jahr emittiert werden.

Das anthropogen erzeugte $\mathrm{CO}_{2}$ bleibt nicht vollständig als $\mathrm{CO}_{2}$ in der Atmosphäre, sondern wird durch den natürlichen Kohlenstoffkreislauf in andere Reservoire gebracht. Entscheidend für den $\mathrm{CO}_{2}$-Anstieg ist nur der Anteil der Emission, der in der Luft verbleibt: die air-borne fraction.

Faßt man die Entwicklung anthropogener $\mathrm{CO}_{2}-$ Emissionen während der nächsten 50-100 Jahre ins Auge, so kann der biosphärische gegenüber dem fossilen Eintrag vernachlässigt werden. Die Abschätzung zukünftiger $\mathrm{CO}_{2}$-Emissionsraten muß sich daher an Energie-Szenarien orientieren, die die Chancen der Energieoption Kohle im Zusammenhang mit Entwicklungstendenzen des globalen Energiebedarfes diskutieren. Diese ergeben sich aus dem zeitlichen Verlauf und dem Maß der Koppelung dreier Faktoren: Bevölkerungswachstum, Wirtschaftswachstum und Effizienz des Energieeinsatzes. Energie-Szenarien sind Versuche, diese drei Faktoren auf der Grundlage demographischer, ökonometrischer und technologischer Analysen zeitabhängig zu modellieren.

In den Szenarien der letzten Jahre wurden die Pro- 


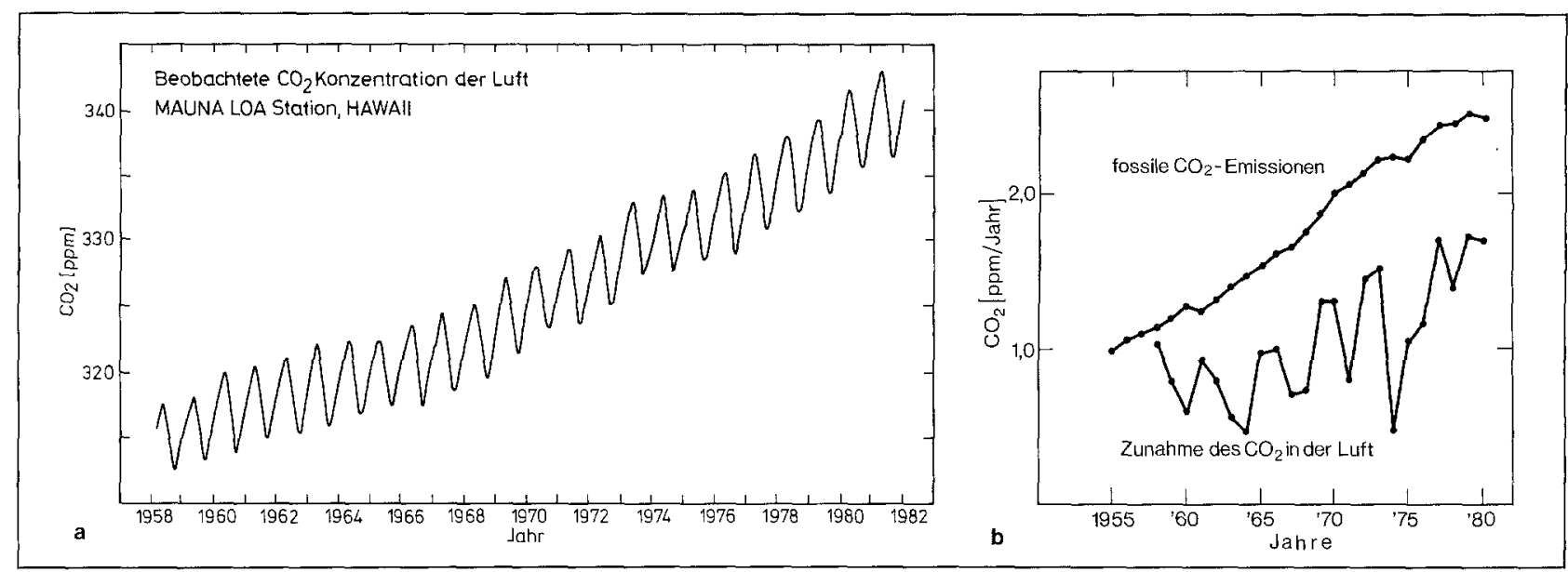

Fig. 1. a) $\mathrm{CO}_{2}$-Konzentration der Luft, Mauna-Loa-Kurve [1], b) der jährliche atmosphärische $\mathrm{CO}_{2}$-Zuwachs seit 1958 im Vergleich mit dem Input fossilen Kohlenstoffs (umgezeichnet nach [1])

gnosen für den erwarteten Weltenergiebedarf kontinuierlich nach unten korrigiert. Heute scheint ein jährliches Wachstum der $\mathrm{CO}_{2}$-Emissionen in den kommenden Jahrzehnten von ca. 2\%, d.h. eine Fortschreibung des Wachstums seit 1973 (Fig. 2), plausibel zu sein. Lovins [4] entwickelte Szenarien energetischen Nullwachstums, ermöglicht durch wesentlich effizienteren Energieeinsatz auf der Grundlage bestehender Technologien.

Für gegebene Werte einer konstant gehaltenen airborne fraction verschiebt sich der Zeitpunkt, zu dem etwa ein $\mathrm{CO}_{2}$-Gehalt von 600 ppmv erreicht sein könnte, entsprechend den angenommenen

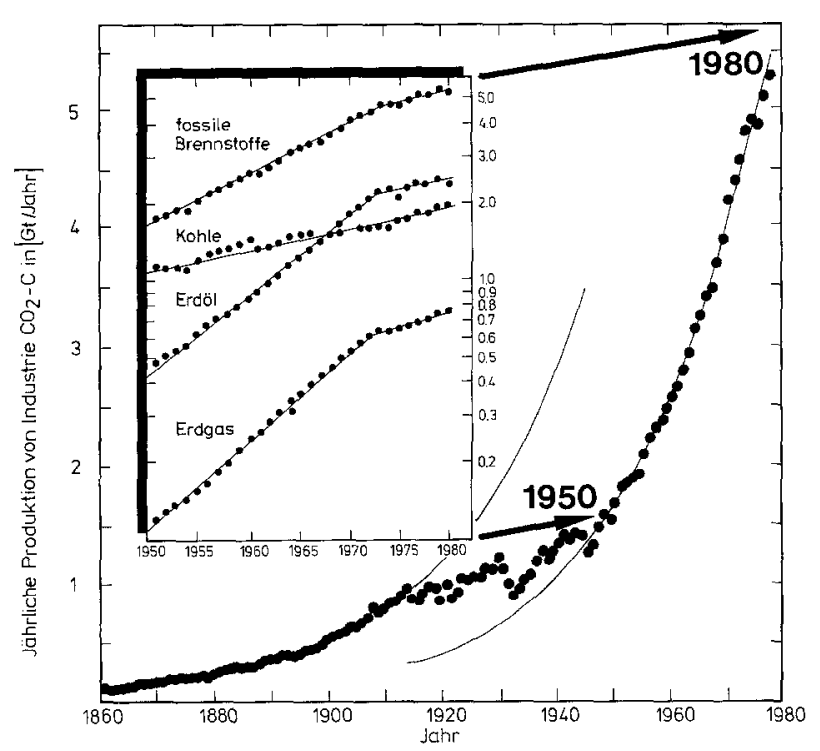

Fig. 2. Verbrauch fossiler Energieträger seit 1860 (umgezeichnet nach [1] und [28])
Wachstumsraten des $\mathrm{CO}_{2}$. Aus Fig. 3 kann man entnehmen, wann 600 ppmv erreicht sein können für beliebige Kombinationen von air-borne fraction und Wachstumsrate von $\mathrm{CO}_{2}$-Emissionen.

\section{Die Verteilung des $\mathrm{CO}_{2}$ im Kohlenstoff-Kreislauf}

Für Klimamodelle ist die zeitliche Entwicklung der air-borne fraction der wichtigste Eingabeparameter, der Energie- und Klima-Szenarien koppelt. Sie ist der atmosphärische Ausdruck der Umverteilung von natürlichem und anthropogenem Kohlenstoff

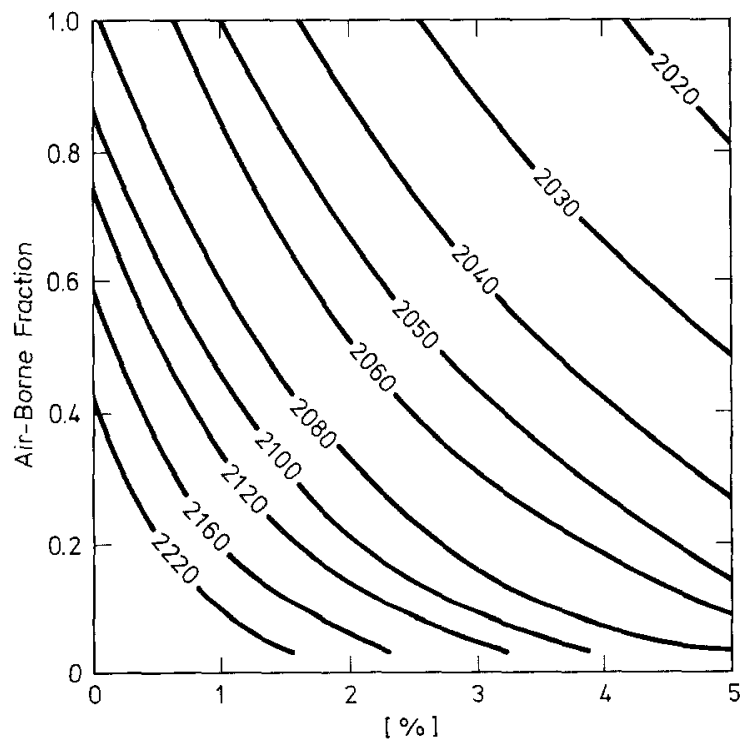

Fig. 3. Jahr, in dem eine Verdopplung des Luft- $\mathrm{CO}_{2}$-Gehaltes erreicht wird, für beliebige Kombinationen des Anstiegs im Verbrauch fossiler Energieträger und der air-borne fraction (nach [28]) 
Tabelle 1. Reservoiregrößen und Flußraten des Kohlenstoffkreislaufs

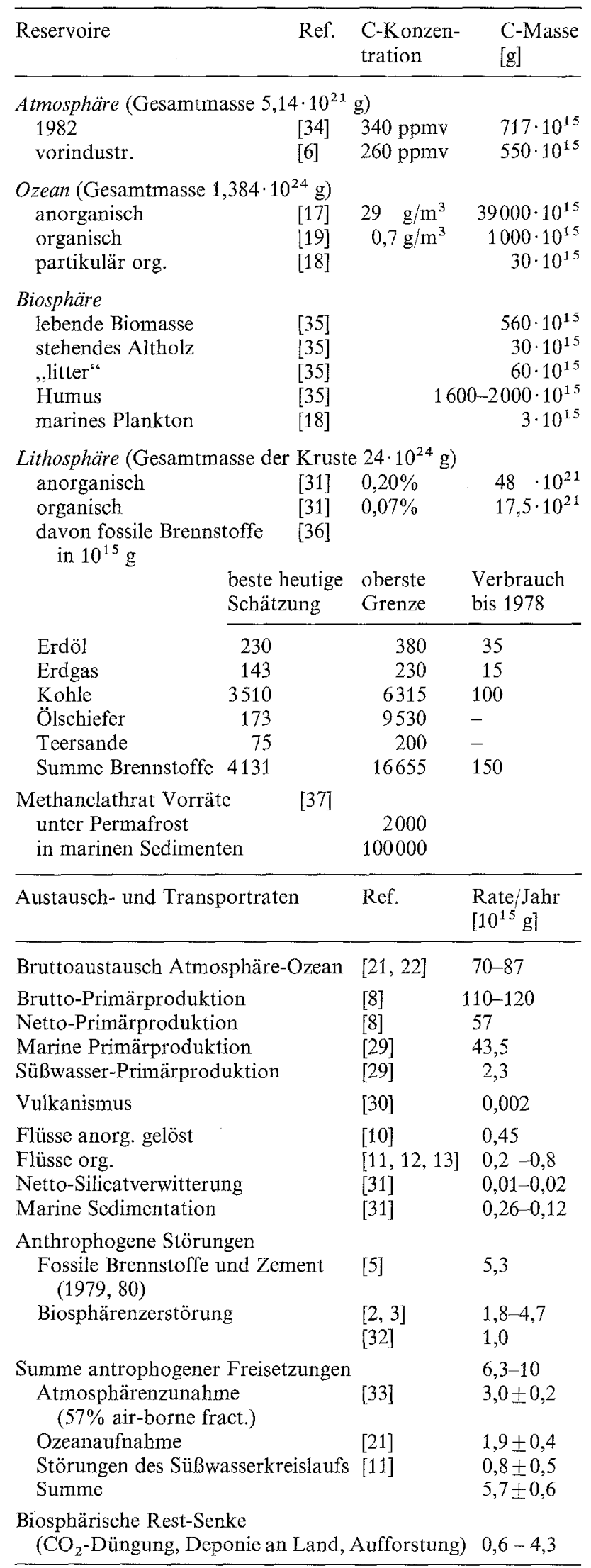

im Kohlenstoff-Kreislauf. Ihre Prognose setzt Kohlenstoff-Kreislauf-Modelle voraus, die in der Lage sind, verschiedene Beobachtungstatsachen unabhängig von freien Parametern zeitabhängig wiederzugeben.

Die Anpassung von Modellen des KohlenstoffKreislaufs an die Meßwerte der Mauna-Loa-Station ist, da die Quellenfunktion des fossilen $\mathrm{CO}_{2}$ bekannt ist [5], möglich durch Variation von drei Größen: 1. vorindustrieller $\mathrm{CO}_{2}$-Gehalt der Atmosphäre, 2. Entwicklung der biosphärischen NettoQuelle und 3. $\mathrm{CO}_{2}$-Aufnahme des Ozeans. Sind zwei dieser drei Größen bekannt, so läßt sich weil die fossile Quelle bekannt ist - die air-borne fraction für den Beobachtungszeitraum berechnen. Zur Zeit können aber alle drei Größen nur in erster Näherung abgeschätzt werden (Tabelle 1).

In Fig. $1 \mathrm{~b}$ ist abzulesen, wie sich die air-borne fraction seit 1956 entwickelt hat. Auf das fossile $\mathrm{CO}_{2}$ bezogen, verblieben im Schnitt 57\% in der Luft, der Rest und der biogene Anteil wurden durch den Kohlenstoff-Kreislauf aus der Atmosphäre entfernt. Bisher konnte der vorindustrielle $\mathrm{CO}_{2}-\mathrm{Ge}$ halt der Luft nicht eindeutig rekonstruiert werden. Neuere Messungen an im antarktischen Eis eingeschlossenen Luftbläschen [6,7] ergeben Werte um 260 ppmv (=550 Gt C). Figur 4 zeigt den globalen Kohlenstoff-Kreislauf im Schema.

\section{Die Biosphäre}

Die Größe der Biosphäre auf den Kontinenten ist nur schwer zu erfassen. Vegetationskarten geben die Verteilung der Vegetationstypen für die hier diskutierten Fragestellungen nicht genau genug wieder und sind z.T. durch die fortschreitenden Rodungen schon veraltet.

Für den $\mathrm{CO}_{2}$-Umsatz zwischen Atmosphäre und Biosphäre ist aber nicht in erster Linie der Gesamtbetrag, sondern die Produktivität der stehenden Biomasse wichtig. Das neueste Produktionsmodell (Assjut-Model) von Aselmann und Lieth [8] basiert auf den im World Atlas of Agriculture von 1969 gegebenen Verteilungen der Vegetationstypen und ergibt eine Netto-Primärproduktion (NPP) von $127 \mathrm{Gt}$ Trockenmasse/Jahr. Umgerechnet in Kohlenstoff sind dies (Faktor 0,45) 57,2 Gt C/a. Die Produktivität ist in diesem Modell nur von Temperatur und Niederschlag abhängig. Treibhaus-Experimente weisen darauf hin, daß Pflanzen auf erhöhte $\mathrm{CO}_{2}$-Angebote mit vermehrter Produktion reagieren. Doch basieren die meisten dieser Befunde auf Experimenten mit Nutzpflanzen. Nach allem, was wir derzeit wissen, nimmt die Biomasse auf der Erde bisher eher ab als zu. Ob trotz 


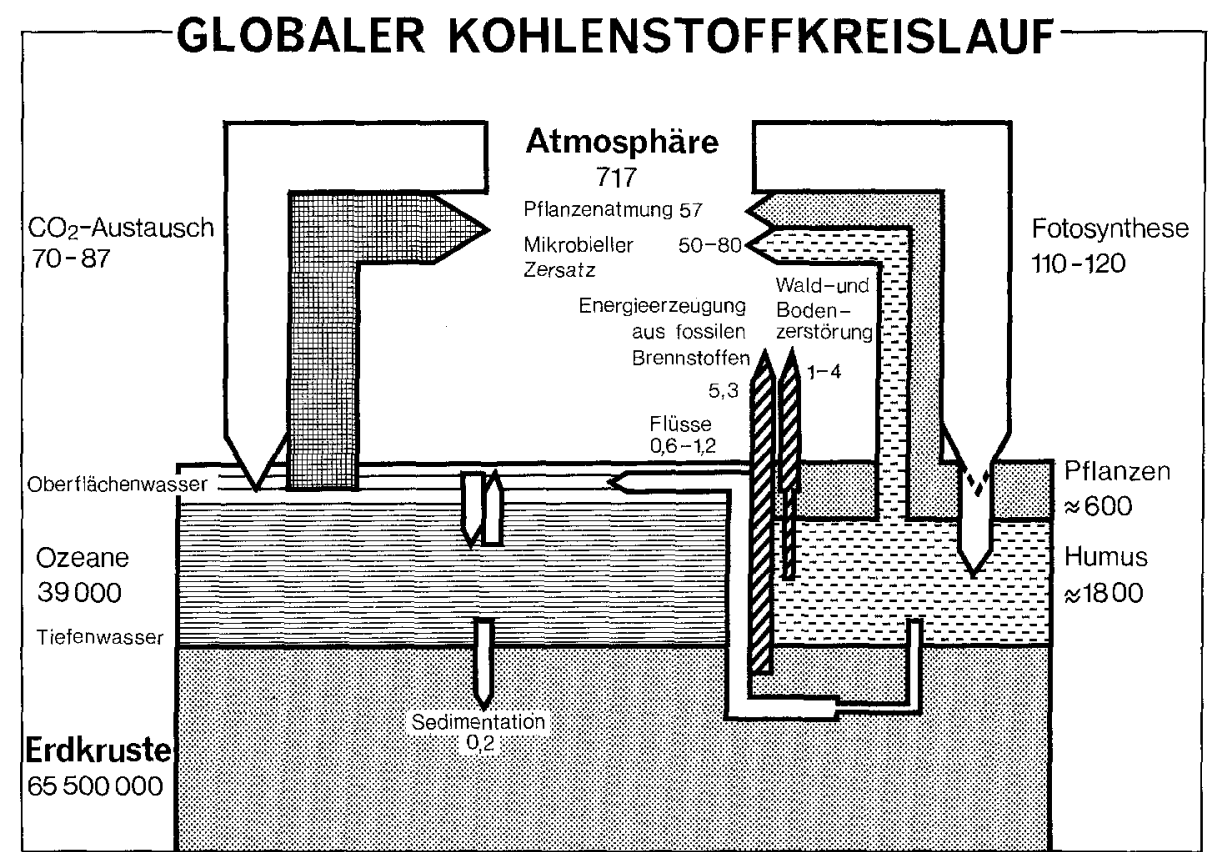

Fig. 4. Schema des KohlenstoffKreislaufs (in Gt C,

Austauschraten in Gt C/Jahr) weltweit reduzierter NPP der Düngeeffekt die Biomassenverluste durch menschlichen Eingriff ausgleicht oder gar übertrifft, ist z.Zt. offen. Möglicherweise wird bei der Rodung von Wäldern und bei der Zerstörung der Böden überhaupt nur ein kleiner Teil der vorhandenen Biomasse $\mathrm{zu} \mathrm{CO}_{2}$ oxidiert. Bei der Brandrodung bleiben in der Regel die Stämme mit ihren großen Ästen stehen. Ein Teil der kleineren Zweige wird zu Holzkohle umgewandelt und gelangt in den Boden. Auch natürliche Waldbrände vernichten nie die gesamte Substanz. Seiler und Crutzen [9] schätzen, daß jährlich 2-4 Gt C durch Brände an die Atmosphäre verloren gehen, die Hälfte des in den betroffenen Gebieten vorhandenen Kohlenstoffs. Dabei werden 0,5-1,7 Gt C Holzkohle, also elementarer Kohlenstoff erzeugt, der sehr resistent gegen Oxidation ist. Überdies geht bei der Bodenzerstörung vermutlich nur ein Teil des Humus durch Oxidation verloren, der Rest dürfte erodiert werden.

\section{Veränderungen in Flüssen, Seen und Randmeeren}

Es ist also zu erwarten, daß Teilmengen des bei der Zerstörung der Biosphäre anfallenden Kohlenstoffs als feste oder gelöste organische Substanz mit den Flüssen verfrachtet werden. Große Mengen werden möglicherweise gar nicht weit transportiert, sondern in Talböden, an Hangfüßen, in Seen und künstlichen Staubecken abgelagert. Ein anderer Teil gelangt mit den Flüssen bis in den Ozean. Der Gesamtransport der Flüsse bis zum
Ozean beträgt etwa $0,45 \mathrm{Gt}$ anorganischen Kohlenstoff und (die Angaben schwanken hier noch sehr stark) ca. 0,4 Gt organischen Kohlenstoff [10-13].

Gegenwärtig versucht eine internationale Arbeitsgruppe im Rahmen des SCOPE/UNEP-Projektes ,Transport of Carbon and Minerals in Major World Rivers“", die jetzt gültigen Transportraten zu messen und die Auswirkungen des Menschen auf den Kohlenstoffhaushalt unserer Flüsse und Seen abzuschätzen [14].

Die Veränderungen des Frischwasser-Systems kann man wie folgt zusammenfassen [11]:

Die Zerstörung der natürlichen Vegetationsdecke und die Ausweitung der Ackerbauflächen erhöht die Erosionsrate um ein Mehrfaches gegenüber dem ungestörten Zustand. Der Hauptteil bleibt auf den Kontinenten. Eine Abschätzung der dabei begrabenen organischen Kohlenstoffmengen fehlt bisher.

Der Transport fester organischer Kohlenstoffverbindungen in den Flüssen ist nur ungenau bekannt. Bis zu 0,5 Gt C/a könnten hier zusätzlich verloren gehen.

Die zusätzliche Ablagerung organischen Kohlenstoffs, z.B. des mitgeführten Humus und des durch Phytoplankton in organische Verbindungen umgewandelten anorganischen Kohlenstoffs in Seen und Stauseen, wird auf $0,25 \mathrm{Gt} \mathrm{C/a}$ geschätzt [15].

Die Zunahme der Ablagerung organischen Kohlenstoffs in den Randmeeren auf Grund der Überdüngung mit durch Flüsse eingeleiteten Stickstoff- 
und Phosphorverbindungen kann $0,1 \mathrm{Gt} \mathrm{C} / \mathrm{a}$ betragen. Hier fehlen noch grundlegende Kenntnisse.

Eutrophierte Seen erreichen während der Phytoplanktonblüten erhebliche $\mathrm{CO}_{2}$-Unterdrücke und werden damit zu kleinen Senken für atmosphärisches $\mathrm{CO}_{2}$.

Erhöhte Zufuhr organischer Substanz in die Flüsse erhöht ihren $\mathrm{CO}_{2}$-Druck erheblich, sie werden $\mathrm{zu}$ kleinen $\mathrm{CO}_{2}$-Quellen. Verschmutzte Flüsse führen mehr freies $\mathrm{CO}_{2}$ als im ungestörten Zustand, meist in der Größenordnung von $10 \%$ des gelösten $\mathrm{HCO}_{3}$. Bei Mischung mit dem Meerwasser wird dieses $\mathrm{CO}_{2}$ durch Lösung mariner Carbonate gepuffert und dauerhaft in $\mathrm{HCO}_{3}$ übergeführt. Die Größe dieser $\mathrm{CO}_{2}$-Senken sollte $0,04 \mathrm{Gt} \mathrm{C/a} \mathrm{nicht}$ überschreiten [16].

Die Summe all dieser kleinen Senken für Kohlenstoff meist biogener Herkunft könnte $1 \mathrm{Gt} \mathrm{C} / \mathrm{a}$ betragen.

\section{Der Ozean und seine Modellierung}

Der Ozean enthält $39000 \mathrm{Gt}$ gelösten anorganischen Kohlenstoff, über $50 \mathrm{mal}$ so viel wie die Atmosphäre [17]. Hinzu kommen $1000 \mathrm{Gt}$ gelösten organischen Kohlenstoffs [18-20]. Verschiedene Prozesse regeln, in welchem Maße und in welcher Zeit $\mathrm{CO}_{2}$-Druckerhöhungen in der Luft sich dem Ozean mitteilen.

Die Geschwindigkeit des Gasaustausches durch die Ozeanoberfläche ist endlich; der Fluß des $\mathrm{CO}_{2}$ wird nach verschiedenen Methoden auf 16-20 mol $\mathrm{CO}_{2} / \mathrm{m}^{2} / \mathrm{a}$ geschätzt $[21,22]$. Daraus folgt eine mittlere Verweilzeit von $\mathrm{CO}_{2}$ in der Atmosphäre von etwa 10 Jahren.

Nach einer Erhöhung des $P_{\mathrm{CO}_{2}}$ in der Luft setzt ein Nettofluß in den Ozean ein, und zwar so lange, bis der $P_{\mathrm{CO}_{2}}$ der Luft wieder im Gleichgewicht mit der Kohlenstoffmenge im Ozeanwasser ist. Dabei ist $\mathrm{zu}$ beachten, daß lediglich $1 \%$ des anorganischen Kohlenstoffs gelöstes $\mathrm{CO}_{2}$ und $\mathrm{H}_{2} \mathrm{CO}_{3}$ ist, $99 \%$ liegen dissoziiert als $\mathrm{HCO}_{3}$ und $\mathrm{CO}_{3}$ vor. Bei Erhöhung des Gesamtkohlenstoffs in Luft und Ozean wird das Gleichgewicht zugunsten der undissoziierten Formen verschoben. Andererseits bedeutet eine Erhöhung des $\mathrm{CO}_{2}$-Luftdrucks $\left(P_{\mathrm{CO}_{2}}\right)$ von $10 \%$ (d.i. $30 \mathrm{ppmv}$ ) eine Zunahme des Gesamt- $\mathrm{CO}_{2}$ im Meerwasser der Deckschicht um nur $1 \%$. Dieser Pufferfaktor wird zudem bei zunehmendem $P_{\mathrm{CO}_{2}}$ ungünstiger.

Außerdem steht nicht das gesamte Wasser des Ozeans mit der Luft in Verbindung. Mehr als 90\% der $1,5 \cdot 10^{9} \mathrm{~km}^{3}$ Wasser sind unter einer warmen, ca. $75 \mathrm{~m}$ mächtigen Oberflächenschicht abge- schlossen, die saisonal erwärmt und durch Stürme gemischt werden kann. Darunter liegt die Thermokline, eine stabile Dichtesprungschicht. Das Tiefenwasser der Ozeane steht nur im Nordatlantik und am Rande der Antarktis mit der Oberfläche in Verbindung. Hier sind die Winter kalt genug, um Oberflächenwasser in die Tiefsee absinken zu lassen. Langsames Aufquellen der Tiefenwässer über die Gesamtfläche der Ozeane und in speziellen Auftriebsgebieten ersetzt das absinkende Tiefenwasser und schließt den Kreislauf. Messungen des ${ }^{14} \mathrm{C}$-Gehaltes der Tiefsee ergaben, daß die mittlere Verweildauer der Wässer unter $1500 \mathrm{~m}$ im Atlantik 275 Jahre, im Pazifik 510, im Indik 250 und im Zirkumpolaren Ozean 85 Jahre beträgt. Die Aufquellraten betragen 4,5 und $10 \mathrm{~m} / \mathrm{a}$ für Atlantik, Pazifik und Indik [23]. Das älteste Wasser im Pazifik ist 1700 Jahre alt; im Schnitt wird der Ozean aber alle 500 Jahre umgewälzt.

Auf Grund dieser Beobachtungen lassen sich charakteristische Zeitskalen definieren. Die warme Oberflächenschicht ist innerhalb von Monaten an atmosphärische Änderungen angeglichen. Das Wasser bis zur Hauptsprungschicht hat eine Einstellzeit von etwa $10 \mathrm{Jahren}$ und das Tiefenwasser eine solche von einigen hundert Jahren.

Man hat in den letzten Jahren viele Modelle vorgeschlagen, um die Speicherfähigkeit des Ozeans numerisch zu modellieren. In den meisten Modellen wird der Ozean in Boxen (Oberflächenschicht, Sprungschicht, Tiefenwasser) eingeteilt, zwischen denen Flußkonstanten, die etwa den besprochenen Zeitkonstanten entsprechen, den Stofftransport vermitteln. Die beste Zusammenstellung dieser Modelle haben Bacastow und Björkström [24] gegeben.

Prinzipieller Nachteil dieser Modelle ist, daß die Pufferfaktoren und Zeitkonstanten nicht a priori bekannt sind. Sie werden in der Regel erst im Zusammenhang mit diesen Modellen aus der gemessenen Verteilung von Tracern $\left({ }^{14} \mathrm{C}\right.$, Tritium) und aus dem fossilen $\mathrm{CO}_{2}$-Input der letzten 100 Jahre bestimmt. Die Prognosefähigkeit dieser Modelle ist demgemäß begrenzt, zumal die Darstellung des Kontinuums ,Ozean“ durch wenige Boxen (maximal 13 wurden gerechnet) nur eine grobe Näherung sein kann.

Am Max-Planck-Institut in Hamburg wird z.Zt. ein Modell entwickelt, das von diesen Restriktionen weitgehend frei ist [25]. Transportiert wird mit einem realistischen dreidimensionalen Strömungsmodell der ozeanischen Zirkulation, $\mathrm{CO}_{2}$ ist lediglich als Tracer in dieses Feld eingebettet. Die Topographie der Kontinente und das gemessene Temperaturfeld der Meeresoberfläche werden berücksich- 
tigt [26]. Der Ozean wird durch Gitterpunkte mit $500 \mathrm{~km}$ Abstand, vertikal in fünf Schichten aufgelöst, dargestellt $(75,150,300,1000$ und $3000 \mathrm{~m})$. Wassermassen werden nicht voneinander abgegrenzt, so daß auch keine expliziten Zeitkonstanten nötig sind. Ein vereinfachtes chemisches System aus den einzelnen marinen $\mathrm{CO}_{2}$-Fraktionen, der Alkalinität und Borat steuert den Gas-Austausch zwischen Meer und Luft. Zur Zeit enthält das Modell noch keine Prozesse, die die Alkalinität ändern könnten, die Alkalinität bleibt konstant. Löslichkeit des $\mathrm{CO}_{2}$ und Dissoziationskonstanten sind
Funktionen der Témperatur. Die einzige prognostische Variable ist damit die Summe des marinen $\mathrm{CO}_{2}$, dessen Transport im Ozean durch die Geschwindigkeitsfelder des Strömungsmodells berechnet wird. Die Randbedingung an der Meeresoberfläche setzt die Oberfläche bei bekannter Alkalinität mit dem vorher errechneten $\mathrm{CO}_{2}$-Druck der Luft ins Gleichgewicht. In einem weiteren Schritt wird die in der Luft eben erzeugte Inhomogenität durch Diffusion weitgehend ausgeglichen. Für die untersuchten langsam veränderlichen Vorgänge wurde ein Zeitschritt von einem Jahr be-

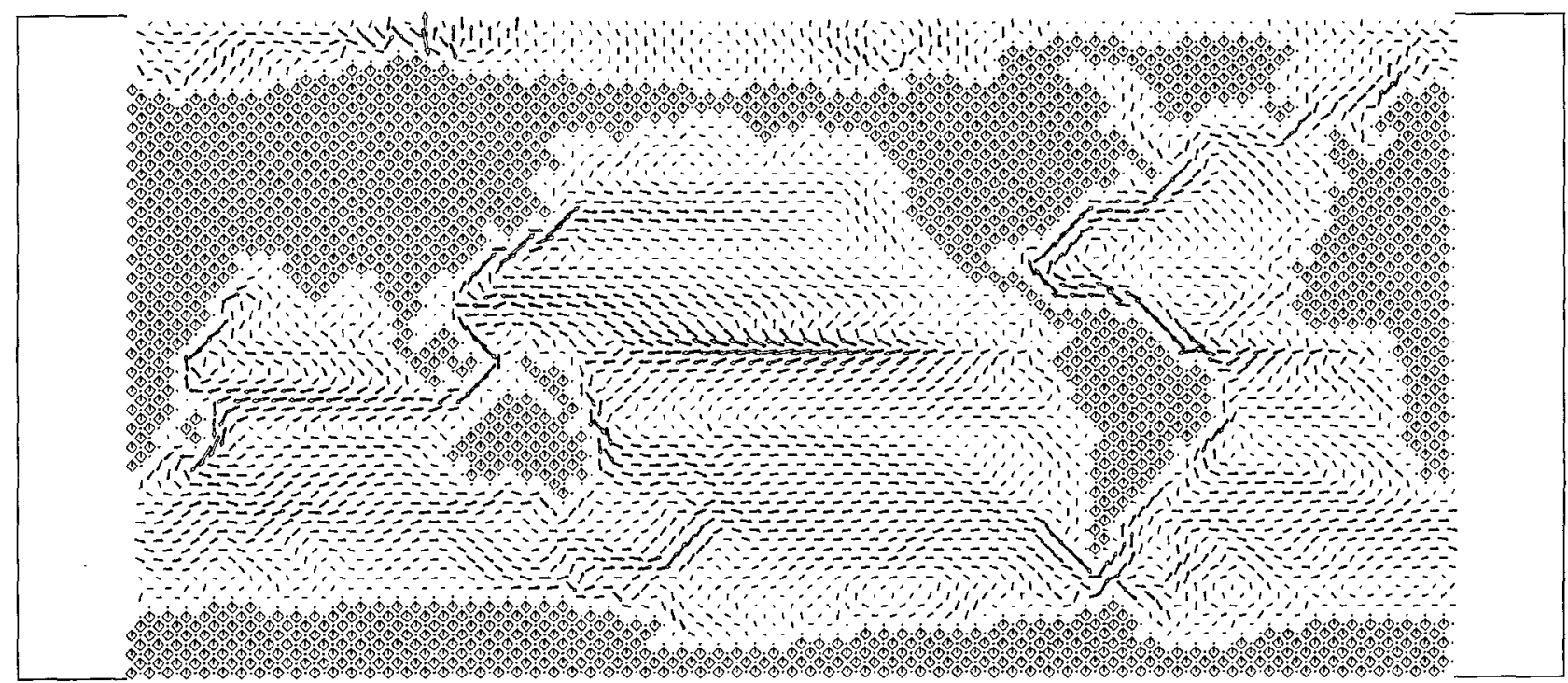

Fig. 5. Strömungsfeld des Ozeanmodells (abs. Geschwindigkeit in $75 \mathrm{~m}$ Tiefe)

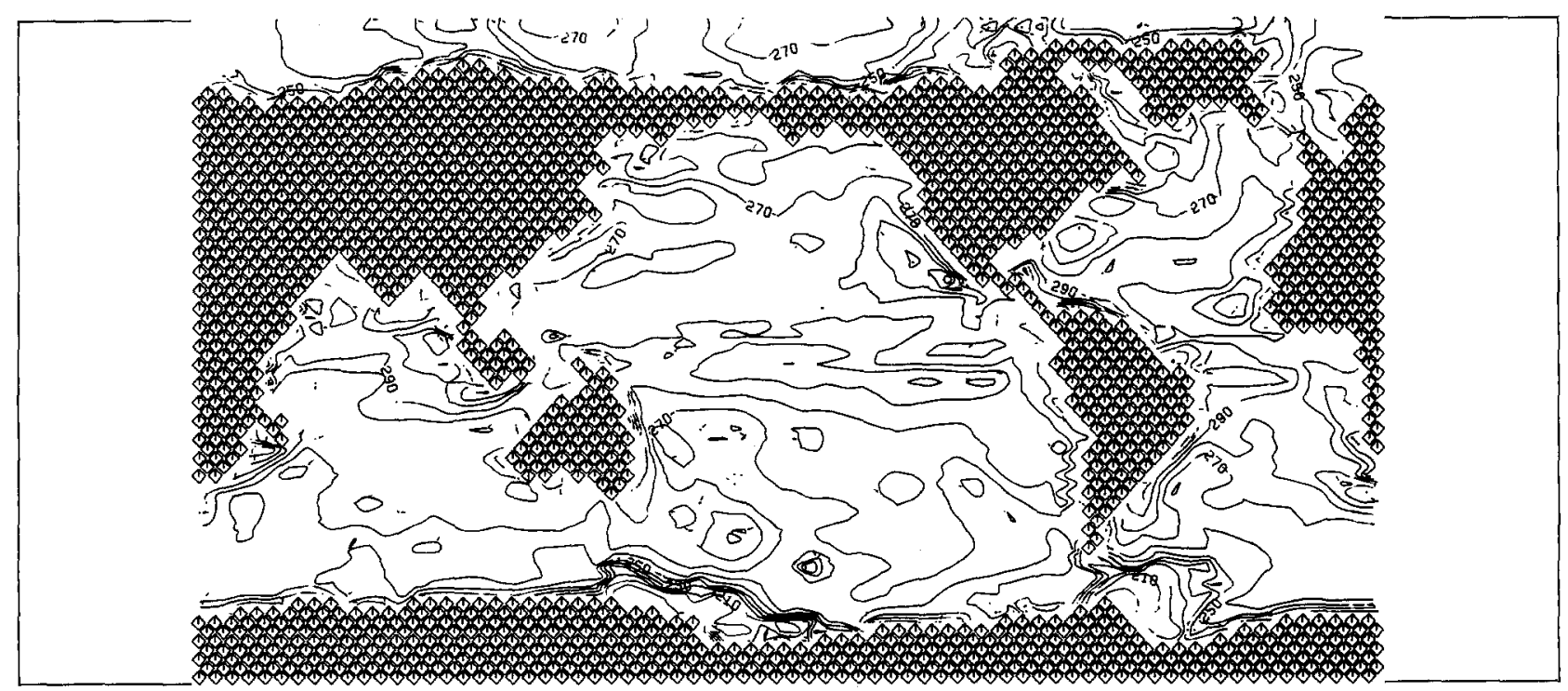

Fig. 6. $P_{\mathrm{CO}_{2}}$ des Ozeanmodells. Oberfläche bei $285 \mathrm{ppm}$ atmosphärischem $P_{\mathrm{CO}_{2}}$ 
nutzt. In dieser Zeitspanne kann mit einer realistischen Gasaustauschrate eine Equilibrierung zwischen Atmosphäre und der obersten Schicht des Modells angenommen werden. Das Modell reagiert naturgemäß empfindlich auf die Vorgabe von Alkalinität und Luft- $P_{\mathrm{CO}_{2}}$ zur vorindustriellen Zeit. Der Einfluß dieser ,Anfangsbedingungen“" wurde in einer Reihe von Experimenten untersucht.

Das Strömungsmodell löst die wesentlichen, akzeptierten Merkmale der ozeanischen Zirkulation hinreichend auf. Figur 5 zeigt die errechnete Oberflächenströmung. Das Strömungsfeld wurde nicht künstlich an Beobachtungen angepaßt. Zur Zeit wird das Strömungsmodell durch Jahresmittelwerte von Wind und Oberflächentemperatur angetrieben. Die starke Winterkonvektion in nördlichen Breiten wird noch nicht durch das Modell wiedergegeben. Die Ergebnisse des $\mathrm{CO}_{2}$-Abtransportes sind daher im Modell noch kleiner als in der Natur zu erwarten. Figur 6 zeigt den $P_{\mathrm{CO}_{2}}$ der Meeresoberfläche, der einem Luft- $P_{\mathrm{CO}_{2}}$ von $285 \mathrm{ppm}$ wie zur vorindustriellen Zeit entspricht. Die sichtbaren Inhomogenitäten entsprechen dem Temperaturfeld. Während regionale Schwankungen der Atmosphäre lediglich wenige ppm ausmachen, sind die Unterschiede auf der Meeresoberfläche weit größer. In Auftriebsgebieten gibt durch Einstrahlung erwärmtes Tiefenwasser ständig $\mathrm{CO}_{2}$ $\mathrm{ab}$, in kalten Polargebieten wird ständig $\mathrm{CO}_{2}$ gelöst.

Drei Experimente, die mit herkömmlichen BoxModellen nicht zu machen sind, seien vorgestellt:

\section{Glazialer Zustand}

Unter Beibehaltung des derzeitigen Strömungsfeldes wurde die Gleichgewichtseinstellung bei um $3{ }^{\circ} \mathrm{C}$ niedrigeren Temperaturen als heute untersucht. Der atmosphärische $P_{\mathrm{CO}_{2}}$ sank dabei von 285 auf 225 ppmv ab. Vergleicht man dies mit den Messungen von Neftel et al. [7], die für das Hochglazial vor 18000 Jahren in Eiskernen Werte von 190-230 ppmv gemessen haben, so liegt der Schluß nahe, daß der größte Teil der $P_{\mathrm{CO}_{2}}$-Zunahme seit der letzten Eiszeit allein durch den Temperaturanstieg zu erklären wäre. Gegen diese Interpretation sprechen jedoch Abschätzungen der globalen Meeresoberflächentemperatur während des Hochglazials (zusammengefaßt in [27]), die eine maximale Änderung von nur $1,5 \pm 1,5^{\circ} \mathrm{C}$ zulassen. $\mathrm{Zu}$ dem würde der $P_{\mathrm{CO}_{2}}$-Abfall fast zur Gänze durch die zunehmende Salinität des Ozeanwassers kompensiert, da die Eiskappen ca. 4,5\% des heutigen Ozeanvolumens festlegten [27].

\section{Effektive Zeitkonstanten}

Die in Box-Modellen fest vorzugebenden Zeitkonstanten können - zumindest in ihrer wirksamen Kombination - vom Modell dynamisch bestimmt werden. Untersucht wurde die Pufferung eines einmaligen, großen $\mathrm{CO}_{2}$-Inputs in die Atmosphäre. Das Abklingen der atmosphärischen Konzentration kann durch Überlagerung nur weniger Exponentialfunktionen beschrieben werden. Die Ab-

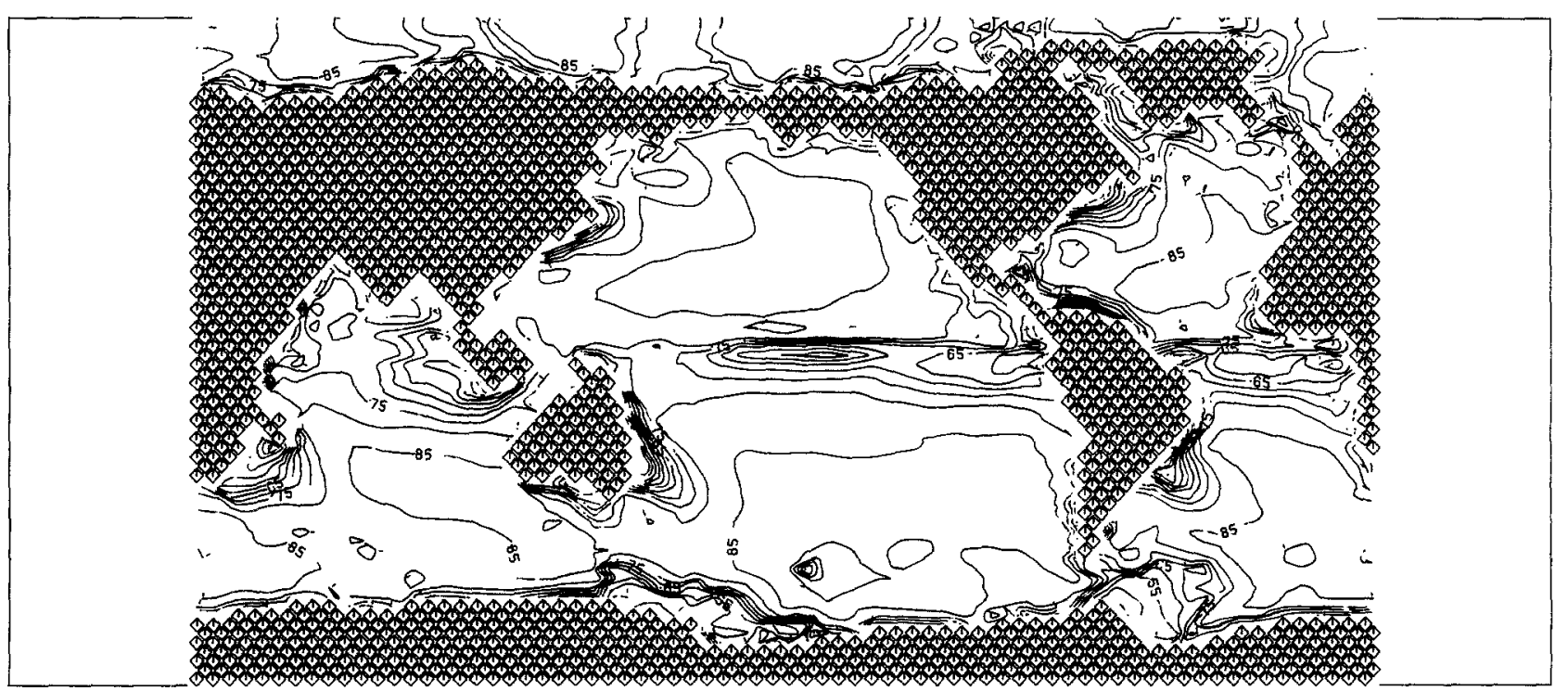

Fig. 7. Netto $P_{\mathrm{CO}_{2}}$-Zuwachs der Ozeanoberfläche zwischen 1860 und 1980 nach Berechnungen des Ozean-Zirkulationsmodells 


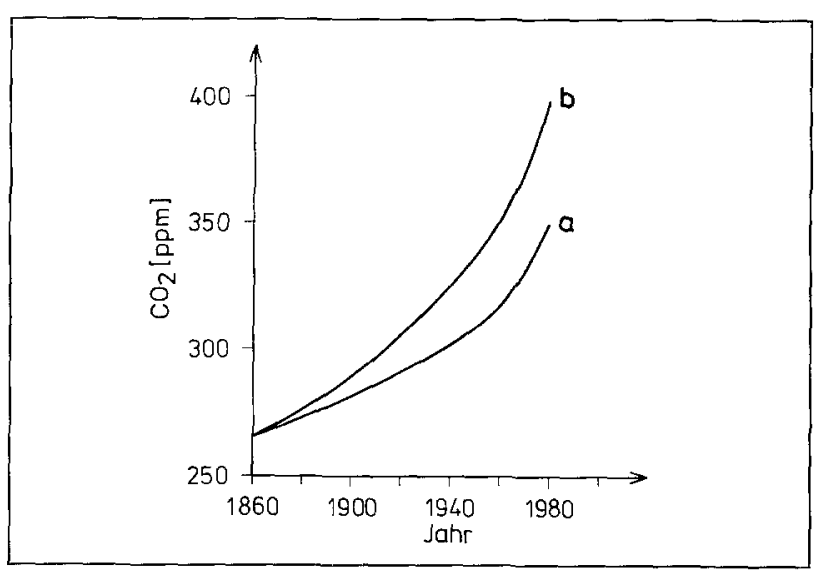

Fig. 8. Vergleich zwischen der Inputfunktion des fossilen $\mathrm{CO}_{2}$ (a) und des im Modell errechneten Atmosphären- $\mathrm{CO}_{2}$-Gehaltes (b)

klingzeiten dieser Funktionen entsprechen den akzeptierten Werten von etwa 1, 10 und 500 Jahren. Sie hängen jedoch auch von der Stärke des einmaligen Inputs ab. Bei einer plötzlichen Verdoppelung des Luft- $P_{\mathrm{CO}_{2}}$ ist die wichtigste Zeitkonstante 160 Jahre; bei einer Vervierfachung wird der $\mathrm{CO}_{2}$-Eintritt in den Ozean aus chemischen Gründen stärker blockiert, die Hauptzeitkonstante erreicht dann 280 Jahre. Diese ,empirischen Greenfunktionen“ stellen einen großen Vorteil gegenüber linearen Box-Modellen dar; sie könnten dazu benutzt werden, eine neue Klasse von einfachen, nichtlinearen Box-Modellen zu konstruieren.

\section{Speicherung von fossilem $\mathrm{CO}_{2}$}

In einem dritten Experiment wurde die Aufnahme von fossilem $\mathrm{CO}_{2}$ für die Zeit von 1860 bis 1980 durch den Ozean berechnet. Als Input dienten die Daten von Rotty [5], erweitert durch einen biogenen Input von $1 \mathrm{Gt} \mathrm{C} / \mathrm{a}$.

Dieser Input entsteht überwiegend auf den Kontinenten nördlich $25^{\circ} \mathrm{N}$. Figur 7 zeigt den errechneten Anstieg des $P_{\mathrm{CO}_{2}}$ an der Meeresoberfläche und Fig. 8 vergleicht den über die Zeit integrierten Input mit dem in der Atmosphäre verbliebenen $\mathrm{CO}_{2}$. Für die letzten 20 Jahre ergibt sich dann eine airborne fraction von $63 \%$.
1. Keeling, C.D., Bacastow, R.B., Whorf, T.P., in: Carbon Dioxide Review: 1982, p. 377 (W.C. Clark, ed.). Oxford Univ. Press 1982

2. Moore, B., et al.: SCOPE Rep. 16, 365 (1981)

3. Houghton, R.A., et al.: Ecol. Monogr. 53, 235 (1983)

4. Lovins, A.B., in: Interactions of Energy and Climate, p. 1 (W. Bach, J. Pankrath, J. Williams, eds.). Dordrecht: Reidel 1980

5. Rotty, R.: J. Geophys. Res. 88 (C2), 1301 (1983)

6. Barnola, J.M., et al.: Nature 303,410 (1983)

7. Neftel, A., et al.: ibid. 295, 220 (1982)

8. Aselmann, I., Lieth, H.: Mitt. Geol.-Paläont. Inst. Univ. Hamburg, SCOPE/UNEP Sonderbd. (im Druck)

9. Seiler, W., Crutzen, P.J.: Climat. Change 2, 207 (1980)

10. Kempe, S.: SCOPE Rep. 13, 317 (1979)

11. Kempe, S.: submitted

12. Meybeck, M.: Am. J. Sci. 282, 401 (1982)

13. Schlesinger, W.H., Melack, J.M.: Tellus 33, 172 (1981)

14. Degens, E.T. (ed.): Transport of Carbon and Minerals in Major World Rivers, Vol. I. Mitt. Geol.-Paläont. Inst. Univ. Hamburg, SCOPE/UNEP Sonderbd. 52 (1982)

15. Mulholland, P.J.: Inland Aquatic Ecosystems and the Perturbed Global Carbon Cycle. ORAN/IEA-817 (M), Inst. for Energy Analysis, Oak Ridge 1981

16. Kempe, S., in: [14], p. 91

17. Bolin, B., et al.: SCOPE Rep. 13, 1 (1979)

18. Mopper, K., Degens, E.T.: ibid. 13, 293 (1979)

19. Mycke, B., Kempe, S., in: RV. Sonne IV Cruise, May, June 1978, Bremerhaven-Panama-Hawaii (S. Kempe, ed.), Final Rep. to the German Res. Council (unpublished)

20. Williams, P.J. LeB., in: Chemical Oceanography, Vol. II, p. 301 (J.P. Riley, G. Skirrow, eds.). London: Academic Press 1975

21. Broecker, W.S., et al. : Science 206, 409 (1979)

22. Peng, T.-H., et a1.: J. Geophys. Res. 84, 2471 (1979)

23. Stuiver, M., Quay, P.D., Östlund, H.G.: Science 219, 849 (1983)

24. Bacastow, R., Björkström, A. : SCOPE Rep. 16, 29 (1981)

25. Maier-Reimer, E., et al.: in Vorbereitung

26. Levitus, S., Oort, H.A.: Bull. Am. Meteorol. Soc. 58, 1270 (1977)

27. Broecker, W.S.: Geochim. Cosmochim. Acta 46, 1689 (1982)

28. Clark, W.C., et al., in: Carbon Dioxide Review: 1982, p. 3 (W.C. Clark, ed.). New York: Oxford Univ. Press 1982

29. de Vooys, C.G.: SCOPE Rep. 13, 259 (1979)

30. Leavitt, S.W.: Environm. Geol. 4, 15 (1982)

31. Kempe, S.: SCOPE Rep. 13, 343 (1979)

32. Olson, J., in: Carbon Dioxide Review: 1982, p. 388 (W.C. Clark, ed.). New York: Oxford Univ. Press 1982

33. Bacastow, R., Keeling, C.D.: SCOPE Rep. 16, 103 (1981)

34. Fraser, P.J., Hyson, P., Pearman, G.I.: Proc. WMO/ICSU/ UNEP Sci. Conf. on Analysis and Interpretation of Atmospheric $\mathrm{CO}_{2}$ Data, Bern 1981, p. 179

35. Ajtay, G.L., Ketner, P., Duvigneaud, P.: SCOPE Rep. 13, 129 (1979)

36. Laurmann, J.A., Rotty, R.M.: J. Geophys. Res. 88 (C2), 1295 (1983)

37. Bell, P.R., in: Carbon Dioxide Review: 1982, p. 401 (W.C. Clark, ed.). New York: Oxford Univ. Press 1981

Eingegangen am 11. August 1983 